\title{
Study on Connection Admission Control Method Based on Wireless
}

\section{Sensor Network}

\author{
Luo Xiao-juan \\ Department of Computer Science, Pingxiang university , PingXiang of JiangXI province , China
}

Keywords: Wireless Sensor Network, Connection Admission Control, Wavelet Transfer, Effective Bandwidth, Overflow

\begin{abstract}
In order to solve the overflow of aggregation node in wireless sensor network, a novel connection admission control method (Connection Admission Control based on Wavelet Transform, CACWT) is proposed. Based on the burst characteristic of wavelet transform reducing network service flow, border of effective bandwidth and overflow probability are derivated. Meanwhile, a simulation test with NS2 was conducted to probe the relationship between effective bandwidth and buffer zone. Compared with other traditional algorithms, CACWT has better suitability.
\end{abstract}

\section{Introduction}

Wireless sensor network, one of the supporting technologies of the Internet of things, is constructed by a wide range of low-cost micro sensor nodes, collaboratively accomplishing the task of collecting, transmission and disposing of perceptual information in the deployment area. It has many characteristics that differ from the traditional wireless network, and can be widely used in many fields such as military affairs, target tracking, environmental monitoring, medical aid, space exploration, precision farming, industrial automation and so on, which has received widespread attention from the industry and academia.

With the fast popularization of wireless sensor network, how to gurantee the performance of service flows has increasingly become a key issue of concern. Admission control is the key technology to ensure the optimizaion of the performace of service flows, it is formed by three parts: service flow description, admission criteria and measuring process. A typical example of the service flow description mechanism is token bucket, and admission criteria is mainly of bandwidth constraint, equivalent capacity and sum-rate, while measuring process usually adopts time window, point sampling and exponential averaging.

In the process of carrying out the connection Admission Control, the network, based on the features of network resources and service flows, decides whether to accept the connection request of the user. Considering such a point, academics both at home and abroad have proposed quite a few evaluating algorithms and evaluation criterions, such as connection admission control based on parameter, connection admission control of end to end statistical model, the dynamic bandwidth reserved mechanism and flow admission control based on distributed measurement, adaptive measurement-based admission control and so on.

A novel connection admission control method is put forward based on the fractal character of service flow. In this method, the burst characteristic of service flow is reduced by wavelet transform at first, and utilizes Fractional Brownian Motion provides that aggregation node of the border of 
effective bandwidth and overflow probability, then a simulation was conduct to deeply analysis the performance conditions of connection admission control.

\section{Introduction of model}

All the nodes in a wireless sensor network (its topological structure shown in Figure 1) are arranged in the same wireless Ad hoc network. Supporting node $\mathrm{K}$ as aggregation node, the rest nodes $\mathrm{N}$ will be data source nodes or relay nodes. In order to copy with the phenomenon of network congestion in time, the performance of network at node $\mathrm{K}$ need to be evaluated. Meanwhile, recently study shows, actual service flows have fractal character and self-similar character. Thus, it needs to combine with this character to build connection admission control method of service flows of node $\mathrm{K}$.

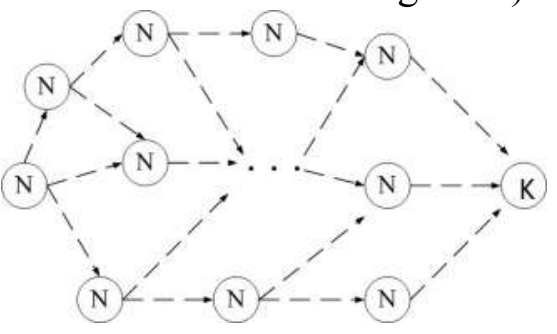

Fig. 1 topological structure of wireless sensor network

The representative method of describing the fractal character of service flow is Fractional Brownian Motion(Fractional Brownian Motion, FBM), defined as:

$$
A(t)=m t+\sqrt{a m} B_{H}(t)
$$

While, $A(t)$ is the aggregating service flows within the moment of time $t, \alpha$ shows as a coefficient of variation, $m$ shows as average arrival rate, $H$ shows as self-similarity $(0<\mathrm{H}<1), B_{H}(t)$ is Norm Fractional Brownian Motion.

Supposing system buffer zone $b$ approach to infinite, if the service rate at node $\mathrm{k}$, i.e. bandwidth $\lambda$ is greater than the average arrival rate $m$ of service flows, the length $\mathrm{Q}$ at node $\mathrm{k}$ needs to satisfy:

$$
\mathrm{Q}(\mathrm{t})=\sup _{\mathrm{s} s \mathrm{t}}(\mathrm{A}(\mathrm{t})-\mathrm{A}(\mathrm{s})-\lambda(\mathrm{t}-\mathrm{s}))
$$

The distribution of the length can be marked as:

$$
\mathrm{P}\{\mathrm{Q}(\mathrm{t})>\mathrm{q}\} \geq \bar{\Phi}\left(\frac{(\lambda-\mathrm{m})^{\mathrm{H}} \mathrm{q}^{1-\mathrm{H}}}{\mathrm{k}(\mathrm{H}) \sqrt{\mathrm{am}}}\right)
$$

And, $\bar{\Phi}()$ is residual function, $k(H)=H^{H}(1-H)^{1-H}$

Supposing the overflow probability at node $\mathrm{K}$ is $\eta$, according to the proposed large deviation theory of document[18], the length of infinite buffer greater than the formula of probability calculation b shall satisfy:

$$
\eta=\mathrm{P}(\mathrm{Q}(\mathrm{x})>\mathrm{b})=\mathrm{e}^{-\frac{(\lambda-\mathrm{m})^{\mathrm{H}} \mathrm{b}^{2-2 \mathrm{H}}}{2 \mathrm{amH}^{2 \mathrm{H}}(1-\mathrm{H})^{1-\mathrm{H}}}}>0
$$

When average arrival rate $\mathrm{m}$ of service flow at node $\mathrm{K}$ is greater than service rate $\lambda$, node $K$ will overflow. The upper bound of overflow probability $P(X(t)>b)$ needs to satisfy:

$$
\left\{\begin{array}{l}
\mathrm{P}(\mathrm{X}(\mathrm{t})>\mathrm{b}) \leq \mathrm{P}\left(\mathrm{m}+\sqrt{\mathrm{am}} \mathrm{B}_{\mathrm{H}}(1)>\lambda\right)=\eta \\
\mathrm{p}\left(\mathrm{B}_{\mathrm{H}}(1)>\frac{\lambda-\mathrm{m}}{\sqrt{\mathrm{am}}}\right)=\mathrm{e}^{-\frac{(\lambda-\mathrm{m})^{2}}{2 \mathrm{am}}}
\end{array}\right.
$$

Then, the upper bound of bandwidth $\lambda_{u p}$ at node $\mathrm{K}$ is : 


$$
\lambda_{\text {up }}=\mathrm{m}+\sqrt{-2 \mathrm{am} \ln \eta}
$$

Meanwhile, when the buffer $b$ is large enough, the lower bound of bandwidth $\lambda_{\text {down }}$ at node $\mathrm{K}$ is:

$$
\lambda_{\text {down }}=\mathrm{m}+\mathrm{b}^{\frac{1-\mathrm{H}}{\mathrm{H}}}\left(\mathrm{k}(\mathrm{H}) \sqrt{-2 \mathrm{M}_{\mathrm{n}+1} \phi_{\mathrm{n}+1} \ln \eta}\right)^{\frac{1}{\mathrm{H}}}
$$

\section{Connection Admission Control Method}

According to the above-derived result of upper and lower bound of bandwidth at node $\mathrm{K}$ (shown as the formula (5) and (7)), one corresponding admission connection control method based on the fractional service flow is to be built. Supposing there exist $\mathrm{n}$ service flows with interdependence and the same parameters $\mathrm{H}$ at node $\mathrm{K}$ in system, as it is know from fractional characteristics, for those independent service flows with the same parameter $\mathrm{H}$, the property of service flows after aggregation remains its parameters unchanged. When a new service flow with the same parameter $\mathrm{H}$ arrives, node $\mathrm{K}$, based on the bandwidth required by $\mathrm{n}+1$ service flows, compares with the system capacity, and if node $\mathrm{K}$ can provide required bandwidth, it will attend the request for connection, or reject. It is supposed to achieve such a result, i.e. the connected average arrival rate of $\mathrm{n}$ service flows after aggregation as $M_{n}$, coefficient of variation as $\phi_{n}$, self-similarity as $\mathrm{H}$. If node $\mathrm{K}$ allows the new arrival service flow, its attributes after aggregation needs to satisfy:

$$
\left\{\begin{array}{l}
M_{n+1}=M_{n}+m \\
\phi_{n+1}=\frac{M_{n} \phi_{n}+m a}{M_{n+1}}
\end{array}\right.
$$

when the buffer size is $b$, the required bandwidth for aggregation flow is $\lambda$ )

$$
\lambda=\mathrm{M}_{\mathrm{n}+1}+\mathrm{b}^{\frac{1-\mathrm{H}}{\mathrm{H}}}\left(\mathrm{k}(\mathrm{H}) \sqrt{-2 \mathrm{M}_{\mathrm{n}+1} \phi_{\mathrm{n}+1} \ln \eta}\right)^{\frac{1}{\mathrm{H}}}
$$

After connecting, the total required bandwidth at node $\mathrm{K}$ is $\lambda_{n+1}$.

$$
\lambda_{\mathrm{n}+1}=\mathrm{M}_{\mathrm{n}+1}+\left(\lambda_{\mathrm{n}}-\mathrm{M}_{\mathrm{n}}\right)\left(\frac{\mathrm{M}_{\mathrm{n}+1} \phi_{\mathrm{n}+1}}{\left(\mathrm{M}_{\mathrm{n}} \phi_{\mathrm{n}}\right)}\right)^{\frac{1}{2 \mathrm{H}}}
$$

If $C_{n+1}$ is less than the actual network bandwidth at node $\mathrm{K}$, the new arriving service flow can be accepted, or rejected. Based on the above description, here is the accurate calculation of admission connection control method at node K:

Step 1 For a certain moment $t$, firstly calculate the attributes of $\mathrm{n}$ service flows at node $\mathrm{K}$ : the average arrival rate $M_{n}$, coefficient of variation $\phi_{n}$, self-similarity co-efficiency $\mathrm{H}$, and the distributed bandwidth is $\lambda_{n}$.

Step 2 At the moment $t+1$, when one new service flow requests for connection, its average arrival rate is $\mathrm{m}$, coefficient of variation is $\alpha$, self-similarity coefficient is $H$, according to the formula (8), then depicts the nature of system after aggregation;

Step 3 calculate the bandwidth $\lambda$ needed by aggregation flow according to the formula (8).

Step 4 If the rest capacity at node $\mathrm{K}$ can satisfy the need of bandwidth $\lambda$, the service flow will be allowed, or rejected.

Step 5 At the moment $t+1$, when one connected flow requests for rescinding, recalculation as per formula (11) of the attributes of aggregation flow is required:

$$
\left\{\begin{array}{l}
M_{n-1}=M_{n}-m_{n} \\
\phi_{n-1}=\frac{M_{n} \phi_{n}-m_{n} \phi_{n}}{M_{n-1}}
\end{array}\right.
$$

Step 6 Skip back to step1 and re-calculate the required bandwidth of the service flow to arrive at the next moment until to the end.

But, due to the strong burst characteristics the actual service flow has, it makes the nodes have no effective suitability during the processing, while wavelet transforms as an effective tool for 
depicting fractal characteristics and smoothing burst. Therefore, this thesis, based on the improvement of wavelet's transforming to the above admission control algorithm, puts forward a new depicting method(Connection Admission Control based on Wavelet Transform, CACWT) . Its basic idea is: firstly transform the wavelet for the arrival service flow to reduce its strong burst characteristic, then calculate the parameters when combining the transformed service flow with the connected service flow, and get the required bandwidth. At the same time, to compare the rest bandwidth at node $\mathrm{K}$ to see whether it can satisfy the requirements, and if yes, the service flow will be accepted, or be rejected. The accurate arithmetic is as follows:

Step 1 For the moment $t$, calculate the attributes of $\mathrm{n}$ service flows at node $\mathrm{K}$ first: average arrival rate $M_{n}$, coefficient of variation $\phi_{n}$, self-similarity $\mathrm{H}$, and the required allocated bandwidth $\lambda_{n}$

Step 2 At the time moment $t+1$, when one new service flow requests for connection, sudden processing to the service flow shall be done based on the formula (12)shown. It adopts the method of Mallat multi-resolution analysis, and it utilizes high-pass filter and low pass filter to disintegrate the service flow, thus gaining the wavelet coefficient $D_{j}$ and approximation coefficient $\mathrm{Aj}$ less than the distinguishability $2^{-j}$, and getting the transformed service flow;

Step 3

$\left\{\begin{array}{l}A_{j+1}=H A_{j} \\ D_{j+1}=G A_{j}\end{array}\right.$

In it, $\mathrm{G}$ is high-pass filter and $\mathrm{H}$ low-pass filter separately, and $\mathrm{j}$ is decomposition level;

Step 4 For the wavelet coefficient $D_{j}$ and approximation coefficient $\mathrm{Aj}$ gained, restructure the service flow according to the formula (3) to reduce its strong burst.

$$
A_{j}=H^{*} A_{j+1}+G^{*} D_{j+1}
$$

And $H^{*}, G^{*}$ is the dual operator of $\mathrm{H}, \mathrm{G}$ respectively

Step 5 calculate the average arrival rate $m$, coefficient of variation $\alpha$, self-similarity is $H$ of the service flow after transforming, and depict the attributes of the aggregated system according to the formula(8).

According to the formula(8), calculate the needed bandwidth $\lambda$ of aggregation flow.

If the rest capacity can satisfy the need of bandwidth $\lambda$ at the node $K$, the service flow will be allowed, or rejected.

Step 6 When one connected flow requests for rescinding at the moment $t+1$, re-calculating the attributes of aggregation flow based on formula (11) is required.

Step 7 Skip back to step1, re-calculating the required bandwidth of the service flow to arrive at next moment, until to the end.

\section{Mathematical Simulation}

Aiming at Admission Connection Control Method CACWT established based on the above, a network simulation model incorporated with NS2 is to be built for verification.

Totally, there are 30 designed data source nodes or $\mathrm{n}$ numbers of relay node in the simulation model, with the node buffer size of 64 packets, buffer size of node K $2048 \mathrm{~kb}$, link capacity of $1024 \mathrm{~kb}$, data packet of 256Byte, a delay of $20 \mathrm{~ms}$, average packet arrival rate of 32 packets $/ \mathrm{s}$ and coefficient of variation $a=23.2$.

Meanwhile, to depict the fractal characteristics of actual service flow, it adopts Fractional Gaussian Noise (Fractional Gaussian Noise, FGN) model to produce the service flow with fractal characteristics at all data source nodes, to achieve a transmitting rate of $32 \mathrm{bps}$ and fractal parameter $H=0.92$. 
In the Admission Connection Control Method CACWT proposed in this thesis, the wavelet basis adopted in the transforming of wavelet is Haar, and the decomposition level is 12 .

In order to compare the advantage of the CACWT method, the traditional effective Bandwidths (EB, Effective Bandwidths) and the Upper Bound of Cell Loss Probability (UBCLP, Upper Bound of Cell Loss Probability) are compared, with the results shown as in figure 2.

Under the condition above, calculate the upper and lower bound of the gained bandwidth of node $\mathrm{K}$ first. Simulating analysis shows the corresponding effective bandwidth of CACWT conforms to the calculating results of the smallest bound while EB a nd UBCLB differ a lot.

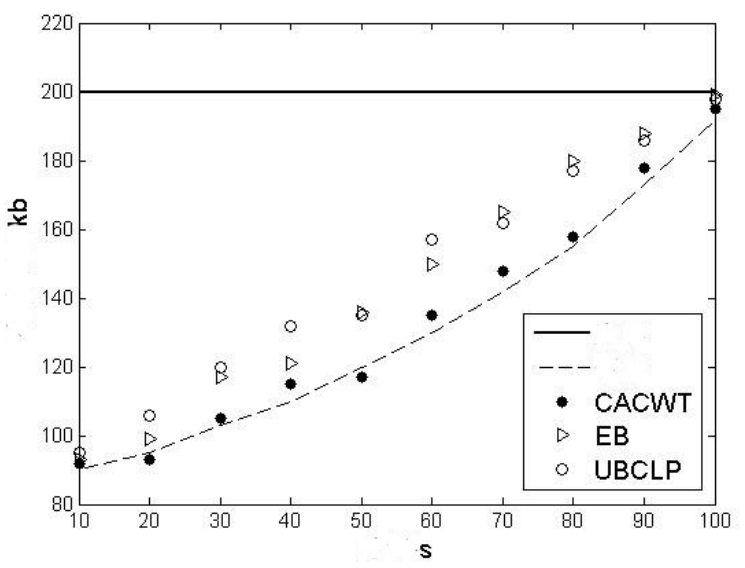

Fig. 3 algorithm comparison

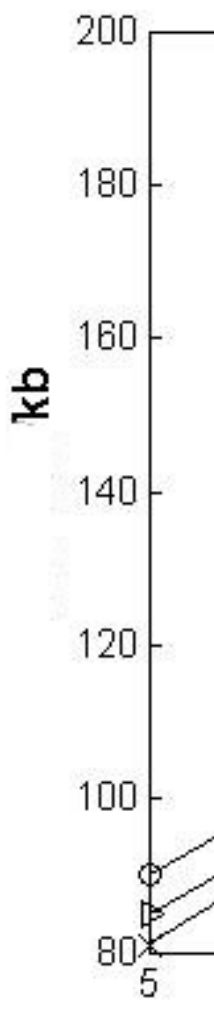

Then, in order to study the performance of the CACWT deeply, here the key factors affecting its performance are adjusted.

Figure 3 shows the relationship between the effective bandwidth and buffer $b$ under the different fractal parameter $\mathrm{H}$

Concluded from Figure 3, with the increase of the buffer, the effective bandwidth will be in downtrend.

And when the buffer is tiny(such as below $500 \mathrm{~kb}$ ), the bigger of the fractal parameter, the smaller of the corresponding effective bandwidth, while the buffer is larger(such as higher than $500 \mathrm{~kb}$ ), the smaller of the fractal parameter $\mathrm{H}$, the smaller of the corresponding effective bandwidth.

The reason for sudden burst lies in the fact that the bounded buffer of the nodes have a large effect on fractal characteristics of service flow of nodes. When buffer overflows, it will interrupt the the later arrival service flow and leads to the occurrence of sudden burst in performance.

Figure 4 shows the relationship between the effective bandwidth and coefficient of variation $\alpha$ under different fractal parameters $\mathrm{H}$. 
With the increasing of the coefficient of variation, the effective bandwidth is also on the rise. But under the same coefficient of variation, the greater of the fractal parameter $\mathrm{H}$, the wider of the effective bandwidth is. It illustrates the coefficient of variation has no effect on effective bandwidth of the sudden burst.

\section{Conclusion}

Aiming at the performance of the service flow of aggregation node and the possible congestion phenomenon in wireless sensor network, this thesis, combining with wavelet transforming, makes some improvements in admission connection control method and defines the upper and lower bounds of overflow probability. And by simulation test with NS2, it makes a deep study on the relationship between effective bandwidth and buffer. By contrasting the traditional Equivalent Bandwidth to the Upper Bound of Cell Loss Ratio, the result shows CACWT has good suitability.

\section{Rreference}

[1] Chouhan S, Bose R, Balakrishnan M. Integrated energy analysis of error correcting codes and modulation for energy efficient wireless sensor nodes[J]. IEEE Transactions on Wireless Communications, 2009, 8(10): 5348-5355.

[2] Vieira F H T, Lee L L. Adaptive wavelet-based multifractal model applied to the effective bandwidth estimation of network traffic flows[J]. IET Communications, 2009, 3(6): 906-919.

[3] Cheng Z L, Burchard A, Liebeherr J. A network calculus with effective bandwidth[J]. IEEE/ACM Transactions on Networking, 2007, 15(6): 1442-1453.

[4] LiJingbo, ZhangGuangsheng, XuZhenyang, DouWenhua. Based on the measurement of self-similarity admission control algorithm research[J]. Hunan University Journal(JCR-SCI) 2010, 37(2): 64-69

[5] PangBin, ShaoHuairong,GaoWen. Distinguish between service network admission control scheme based on measuring the design and application[J],Jurnal of Computers 2003, 26(3):

[6] ChenYanping, Wang Huiqiang, FenGuangsheng, GaoYulong. For statistical time scales of QoS gurantee admission control algorithm[J] Jounal on Communications 2011, 32(11):

[7] Paolo G, Gabriella S. Resource allocation and admission control for the provisioning of quality of service in networks of static priority schedulers[J]. Computer Networks, 2009, 53(2): 231-243.

[8] Zeng Juling, JiangLisi. Based on cross-layer adaptive reserved bandwidth and multiple QoS guarantee of EDCA flow admission control[J] computer science 2012, 39(8):

[9] Weeraddana P C, Codreanu M, Latva-Aho M. Resource allocation for cross-layer utility maximization in wireless networks[J]. IEEE Transactions on Vehicular Technology, 2011, 60(6): 2790-2811.

[10]Norros I. On the use of fractional Brownian motion in the theory of connectionless networks [J]. IEEE Journal on Selected Areas in Communication, 1995, 13(6): 953-962. 